\title{
POTENCIAL PRODUTIVO DE MADEIRA E PALMITO DE UMA FLORESTA SECUNDÁRIA DE VÁRZEA BAIXA NO ESTUÁRIO AMAZÔNICO ${ }^{1}$
}

\author{
Michelliny de Matos Bentes-Gama², José Roberto Soares Scolforo ${ }^{3}$ e João Ricardo Vasconcellos Gama ${ }^{4}$ \\ RESUMO - Este estudo teve como objetivos analisar a estrutura arbórea e valorar uma floresta secundária de várzea \\ baixa. A área de estudo está localizada na propriedade florestal da Exportadora de Madeiras do Pará Ltda. - EMAPA, \\ município de Afuá, Estado do Pará. A área inventariada corresponde a 12,5 ha, onde foram medidos todos os espé- \\ cimes arbóreos com DAP $\geq 15,0 \mathrm{~cm}$. Verificou-se a ocorrência de 73 espécies, que totalizaram 357,7 indivíduos/ha \\ e área basal de $23,4 \mathrm{~m}^{2} / \mathrm{ha}$. As espécies mais importantes do ambiente estudado foram Virola surinamensis, Symphonia \\ globulifera, Eschweilera coriacea, Pentaclethra macroloba e Astrocaryum murumuru. Entre os grupos de uso foi \\ verificado que as espécies não-comerciais apresentaram o maior número de toras comercializáveis $(24,3$ toras/ha), \\ seguidas das espécies comerciais (16,2 toras/ha) e das potenciais (12,9 toras/ha). A receita potencial de toras/ha foi \\ de US\$ 501,70 e a de palmito/ha foi de US\$ 68,50.
}

Palavras-chave: Análise estrutural, valoração, produto florestal, madeira e palmito.

\section{PRODUCTIVE POTENTIAL OF WOOD AND HEART-PALM IN A SECONDARY LOW FLOODPLAIN FOREST OF THE AMAZON ESTUARY}

\begin{abstract}
The aim of this paper was to analyze tree structure and value a secondary low floodplain forest. The study area is located at EMAPA forest lands, in the county of Afuá, in the state of Pará. The inventoried area presents $12.5 \mathrm{ha}$, where all the tree specimens with $D B H \geq 15.0 \mathrm{~cm}$ were measured. A total of 73 species were identified, corresponding to 357.7 individuals/ha and a $23.4 \mathrm{~m}^{2} / \mathrm{ha}$ basal area, the most important being Virola surinamensis, Symphonia globulifera, Eschweilera coriacea, Pentaclethra macroloba and Astrocaryum murumuru. Among the categories, the non-commercial species showed the highest number of marketable boles $(24.3 / \mathrm{ha})$, followed by the commercial $(16.2 / \mathrm{ha})$ and potential ones $(12.9 \mathrm{boles} / \mathrm{ha})$. The boles and the heart-palms provided a potential income of US\$501.70/ha and US\$68.50/ha, respectively.
\end{abstract}

Key words: $\quad$ Forest structure, valuing, forest product, wood and heart-palm.

\section{INTRODUÇÃO}

Na Região Norte as florestas inundáveis ocupam cerca de $98.000 \mathrm{~km}^{2}$ da Amazônia, dos quais $75.880,8 \mathrm{~km}^{2}$ correspondem às florestas de várzea - $1,6 \%$ da superfície da Amazônia brasileira (Araújo et al., 1986). Embora esse ecossistema apresente uma extensão territorial bem reduzida em relação às áreas de terra-firme, historicamente foi o que primeiro contribuiu com o fornecimento de madeira e produtos não-madeireiros - borracha, sementes oleaginosas e fibras, em escala comercial, e favoreceu o desenvolvimento da pesca e da agricultura na região.

Até a década de 60 a produção agrícola e extrativista da Região Norte era quase que totalmente advinda de várzea. A partir de 1970, quando foram lançados os grandes projetos (Operação Amazônia, POLAMAZÔNIA,

1 Recebido para publicação em 3.5.2001.

Aceito para publicação em 20.6.2002.

2 Pesquisadora da Embrapa Rondônia, Doutoranda em Ciência Florestal da Universidade Federal de Viçosa - UFV, 36571-000 Viçosa-MG, <mbgama@tdnet.com.br>. ${ }^{3}$ Professor do Departamento de Ciências Florestais da Universidade Federal de Lavras, Caixa Postal 37, 37200-000 Lavras-MG, <scolforo@ufla.br>. ${ }^{4}$ Doutorando em Ciência Florestal, DEF-UFV, e <ds40104@correio.ufv.br> 
entre outros) e implementadas as políticas de desenvolvimento direcionadas para a Amazônia, a economia da várzea entrou em declínio, em decorrência de as iniciativas governamentais contemplarem apenas o ecossistema de terra-firme. Apesar disso, Lisboa et al. (1991) afirmam que até o início da década de 90 as várzeas ainda fomentavam cerca de 30 \% da produção madeireira amazônica.

Em se tratando da atividade madeireira nessas áreas, verificou-se que esta ainda permanece arraigada nas bases do extrativismo tradicional, decorrente de uma visão unilateral e cheia de falhas da produção florestal regional, que valoriza apenas algumas espécies madeireiras, persistindo aí a exploração predatória, que inibe a sustentação econômica e ameaça a utilização dos recursos florestais sob uma perspectiva de sustentabilidade.

Entretanto, acredita-se ainda na hipótese de que é possível obter retorno econômico com a atividade madeireira e extrativista em florestas de várzea, inclusive em áreas já exploradas. No intuito de investigar esta premissa, os objetivos deste estudo foram: analisar a estrutura horizontal e vertical e valorar o estoque de exploração madeireiro e palmiteiro de uma floresta de várzea baixa já explorada.

\section{MATERIAL E MÉTODOS}

\section{1. Área de Estudo}

A área de estudo está localizada na propriedade florestal da Exportadora de Madeira do Pará Ltda. - EMAPA, no município de Afuá (009’32"S e 50²3’31"W), Estado do Pará. A empresa dispõe de uma área de 1.200 ha destinada para pesquisas com manejo florestal, dos quais $80 \mathrm{ha}$ são de floresta de várzea baixa que sofreu exploração entre 1955 e 1992, período em que houve exploração intensiva de Virola surinamensis, Carapa guianensis e palmito de Euterpe oleracea (açaí). Os valores médios anuais de precipitação e temperatura correspondem, respectivamente, a $2.500 \mathrm{~mm}$ e $26{ }^{\circ} \mathrm{C}$ (Projeto...-SUDAM, 1984). O solo é do tipo Hidromórfico Glei Pouco Húmico (Vieira, 1988).

\subsection{Amostragem e Coleta de Dados}

A partir do mapa da área e com base nas recomendações da FAO (1974), foram distribuídas sistematicamente 25 unidades amostrais de $20 \times 250 \mathrm{~m}$ ( 0,5 ha), no sentido norte - sul, totalizando uma amostra de 12,5 ha. Em cada parcela foram mensurados todos os indivíduos arbóreos e as palmeiras com diâmetro à altura de $1,30 \mathrm{~m}$ do solo (diâmetro à altura do peito - DAP) $\geq 15 \mathrm{~cm}$, e registrados os nomes regionais e as alturas, total e comercial, de todas as árvores. Foram avaliados o número de toras comerciais de cada árvore com DAP $\geq 45 \mathrm{~cm}$, classificadas em: tora de $1^{\text {a }}$ - (circunferência no meio da tora de $4 \mathrm{~m}$ de comprimento, com casca) $\mathrm{C} \geq 150 \mathrm{~cm}$; tora de $2^{\mathrm{a}}-$ $130 \mathrm{~cm} \leq \mathrm{C}<150 \mathrm{~cm}$; tora de $3^{\mathrm{a}}-110 \mathrm{~cm} \leq \mathrm{C}<130 \mathrm{~cm}$; e o número de árvores de Euterpe oleracea em idade de fornecer palmito comercializável no local de estudo, no caso indivíduos a partir de DAP $\geq 10 \mathrm{~cm}$, classificados em fornecedor de palmito de $1 \underline{a}-\mathrm{DAP} \geq 15 \mathrm{~cm}$ e fornecedor de palmito de $2^{\mathrm{a}}-10 \mathrm{~cm} \leq \mathrm{DAP}<15 \mathrm{~cm}$.

Foi realizada também a classificação das espécies de acordo com sua categoria de uso na região: comerciais - espécies comercializadas no mercado nacional e internacional; potenciais - espécies comercializadas no mercado local e regional; e não-comerciais - ainda não aceitas pelo mercado local. Para classificação das espécies por grupo ecológico utilizou-se a proposta de Oliveira-Filho (1994), adaptada de Swaine \& Whitmore (1988), juntamente com as observações de campo no momento da coleta dos dados e revisões bibliográficas, categorizandose as espécies em: pioneiras, clímax exigentes de luz e clímax tolerantes à sombra. Os dados foram coletados no período de dezembro de 1998 a fevereiro de 1999.

\subsection{Análise dos Dados}

A suficiência amostral da composição florística foi verificada, utilizando-se o procedimento REGRELRP Regressão Linear com Resposta em Platô, do Sistema para Análises Estatísticas - SAEG V.5.0 (Ferreira, 1988; Costa-Neto, 1990; Nappo, 1999). Para o cálculo do padrão de distribuição espacial empregou-se o índice de Morisita (Ludwige \& Reynolds, 1988).

Os índices que caracterizaram a estrutura horizontal e vertical, a quantificação do número de toras por espécie e o número de palmitos foram obtidos através do "software" SISNAT - Sistema de Manejo para Florestas Nativas, desenvolvido pelo professor José Roberto Soares Scolforo, do Departamento de Ciências Florestais da Universidade Federal de Lavras-MG.

Os parâmetros fitossociológicos da estrutura horizontal (densidade, freqüência, dominância e índice de valor de importância) foram calculados segundo Curtis \& Mcintosh (1951), Lamprecht (1964), Mueller-Dombois \& Ellenberg (1974). 
A estrutura vertical foi analisada através da posição sociológica, que permite avaliar a participação das espécies nos diferentes estratos arbóreos. Para determinar os estratos adotou-se a recomendação de Souza (1990), utilizada por Mariscal-Flores (1993), que se baseia no desviopadrão da altura das espécies observadas no inventário florestal.

De posse das variáveis citadas, foi calculado o índice de valor de importância ampliado, de acordo com Finol (1971). Foi calculado também o índice de valor de importância ampliado e econômico (Jardim \& Hosokawa, 1986/ 1987), no qual não foi considerada a regeneração natural, uma vez que o nível de inclusão adotado não contemplou a avaliação deste componente da fitocenose.

Para valoração do potencial produtivo de madeira foi utilizado o preço por classe de tora das espécies comerciais. Já os preços das classes de tora das espécies potenciais e não-comerciais foram definidos por meio da média dos três menores preços por classes de toras (Quadro 1), obtida através da seguinte expressão:

$$
\begin{aligned}
& \mathrm{RPM}_{\mathrm{i}}=\left(\mathrm{P}_{\mathrm{T} 1} \cdot \mathrm{NT}_{\mathrm{T} 1}\right)+\left(\mathrm{P}_{\mathrm{T} 2} \cdot \mathrm{NT}_{\mathrm{T} 2}\right)+\left(\mathrm{P}_{\mathrm{T} 3} \cdot \mathrm{NT}_{\mathrm{T} 3}\right) \mathrm{e} \\
& \mathrm{RPT}=\sum_{\mathrm{i}=1}^{\mathrm{S}} \mathrm{RPM}_{\mathrm{i}}
\end{aligned}
$$

em que $\mathrm{RPM}_{\mathrm{i}}=$ receita potencial de madeira em tora da i-ésima espécie, em US\$; $\mathrm{P}_{\mathrm{Ti}}=$ preço médio por classe de tora da i-ésima espécie, em US\$; $\mathrm{NT}_{\mathrm{Ti}}=$ número de toras da i-ésima espécie, para a j-ésima classe de tora; RPT = receita potencial de toras, em US\$; e $\mathrm{s}=$ número de espécies.

Na valoração do estoque de palmitos foi utilizada a receita potencial de palmito, na qual os indivíduos da palmeira Euterpe oleracea foram avaliados quanto ao número de indivíduos fornecedores de palmitos com as características desejáveis para comercialização local e ao preço por classe de palmito (Quadro 2), utilizando a seguinte expressão:

$$
\mathrm{RP}_{\mathrm{i}}=\mathrm{P}_{\mathrm{CP}} \cdot \mathrm{NP}_{\mathrm{CPi}} \quad \text { e } \quad \mathrm{RPP}=\sum_{\mathrm{i}=1}^{\mathrm{CP}} \mathrm{RP}_{\mathrm{i}}
$$

em que $\mathrm{RP}_{\mathrm{i}}=$ receita potencial da i-ésima classe de palmito, em US\$; $\mathrm{P}_{\mathrm{CPi}}=$ preço da i-ésima classe de palmito, em US\$; $\mathrm{NP}_{\mathrm{CPi}}=$ número de plantas na i-ésima classe de palmito; $\mathrm{CP}=$ número de classes de palmitos utilizadas; e RPP $=$ receita potencial de palmitos, em US\$.
Quadro 1 - Preço médio das toras (PMT) por classe de toras (T) das espécies comercializadas pela EMAPA no município de Afuá, Pará

Table 1 -Medium price of boles (PMT) by bole class (T) of marketable species at EMAPA in Afuá, Pará

\begin{tabular}{|l|r|r|r|}
\hline \multirow{2}{*}{ Espécie } & \multicolumn{3}{|c|}{ PMT (US\$) } \\
\cline { 2 - 4 } Comerciais & \multicolumn{1}{|c|}{$\mathrm{T}_{1}$} & \multicolumn{1}{c|}{$\mathrm{T}_{2}$} & $\mathrm{~T}_{3}$ \\
\hline \multicolumn{1}{|c|}{} & & \\
Bombax munguba & 8,33 & 4,44 & 0,00 \\
Carapa guianensis & 16,67 & 8,33 & 5,00 \\
Cedrela odorata & 22,22 & 13,89 & 8,33 \\
Hymenaea oblongifolia & 17,78 & 10,56 & 6,11 \\
Jacaranda copaia & 11,11 & 6,67 & 4,44 \\
Simarouba amara & 11,11 & 6,67 & 4,44 \\
Virola sp. & 16,67 & 8,33 & 5,00 \\
Virola surinamensis & 16,67 & 8,33 & 5,00 \\
Vochysia maxima & 11,11 & 6,67 & 4,44 \\
\multicolumn{1}{|c|}{ Potenciais } & 10,18 & 5,93 & 2,96 \\
Não-comerciais & 10,18 & 5,93 & 2,96 \\
\hline
\end{tabular}

Em que: $\mathrm{T}_{1}=$ tora de $1^{\underline{a}}-\mathrm{C} \geq 150 \mathrm{~cm} ; \mathrm{T}_{2}=$ tora de $2^{\underline{a}}-130 \mathrm{~cm}$ $\leq \mathrm{C}<150 \mathrm{~cm} ; \mathrm{e} \mathrm{T}_{3}=$ tora de $3^{\mathrm{a}}-110 \mathrm{~cm} \leq \mathrm{C}<130 \mathrm{~cm}$.

Where: $T_{1=}$ bole of $1^{\text {st }}$ class $-C \geq 150 \mathrm{~cm}, T_{2}=$ bole of $2^{\text {nd }}$ class $130 \mathrm{~cm} \leq C<150 \mathrm{~cm}$ and $T_{3}=$ bole of $3^{\text {rd }}$ class $-110 \mathrm{~cm} \leq C$ $<130 \mathrm{~cm}$.

Quadro 2 - Preço médio por classe de palmito comercializado no município de Afuá, Pará

Table 2 - Medium price by class of marketable heart-palm in Afuá, Pará

\begin{tabular}{|l|l|}
\hline \multicolumn{1}{|c|}{ Classe de Palmito } & US\$ \\
\hline Palmito de $1^{\underline{a}}(\mathrm{DAP} \geq 15 \mathrm{~cm})$ & 0,17 \\
Palmito de $2^{\mathrm{a}}(10 \mathrm{~cm} \leq \mathrm{DAP}<15 \mathrm{~cm})$ & 0,11 \\
\hline
\end{tabular}

A receita potencial final foi obtida através da formulação:

$$
R P F=R P T+R P P
$$

em que RPF = receita potencial final, em US\$; e RPT e RPP definidos anteriormente.

\section{RESULTADOS E DISCUSSÃO}

Foram mensurados 4.471 indivíduos, pertencentes a 73 espécies, 58 gêneros e 29 famílias, das quais as que apresentaram a maior riqueza de espécies foram Leguninosae Papilionoideae (8), Leguminosae Mimosoideae 
(6), Leguminosae Caesalpinioideae, Chrysobalanaceae, Guttiferae e Palmae, com cinco cada uma. Leguminosae Papilionoideae e Leguminosae Caesalpinioideae destacaram-se como as famílias mais importantes, com 19 espécies ao todo, como também verificado em Campbell et al. (1986). O valor do índice de diversidade foi $\mathrm{H}^{\prime}=3,35$, superior ao estimado por Anderson et al. (1995) e Macedo (1996) para florestas exploradas de várzea no Estado do Pará.

A suficiência amostral ocorreu a partir da 8 $\mathrm{a}$ parcela, o que significa dizer que uma área de 4 ha, 32 \% da área total amostrada, seria suficiente para amostrar a composição florística da área estudada (Figura 1).

$\mathrm{Na}$ distribuição das espécies pelos grupos ecológicos, todas as espécies comerciais foram classificadas como clímax exigentes de luz; na categoria das potenciais ocorreram 6 espécies clímax tolerantes à sombra, 5 espécies clímax exigentes de luz e 1 espécie do grupo das pioneiras; e entre as não-comerciais foram registradas 18 espécies clímax tolerantes à sombra, 27 espécies clímax exigentes de luz e 7 espécies pioneiras. Observouse que as espécies mais procuradas para comercialização na área estudada enquadram-se no grupo das clímax exigentes de luz. Estes resultados indicam que as operações de corte e derrubada de árvores nesta floresta podem vir a favorecer o estabelecimento e o desenvolvimento das espécies comerciais.

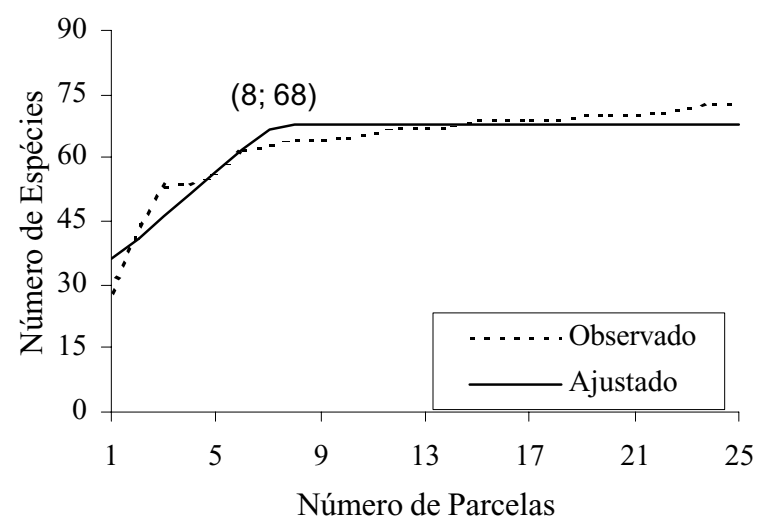

Figura 1 - Curvas acumulativas de espécies em relação ao número de unidades de amostra utilizadas na floresta de várzea baixa, propriedade florestal da EMAPA, município de Afuá, Pará.

Figure 1 - Species cumulative curves in relation to the number of sample plots used in the low floodplain forest at EMAPA forestlands, Afuá, Pará.

R. Árvore, Viçosa-MG, v.26, n.3, p.311-319, 2002
Por meio do índice de Morisita, foi possível observar que a maioria das espécies apresentou um padrão de distribuição agregado (60,3\%), seguido do padrão aleatório (35,3\%); esta mesma tendência foi encontrada por Barros (1986) e Mello (1995). Vale ressaltar que 4,4 \% das espécies não tiveram seu padrão de distribuição calculado, pelo fato de terem ocorrido em apenas uma parcela (Quadro 3).

Analisando a estrutura arbórea foi possível verificar a ocorrência de 357 indivíduos/ha, que representaram uma área basal de $23,4 \mathrm{~m}^{2} / \mathrm{ha}$. Foram observados 331 indivíduos/ha no estoque de crescimento $(15 \mathrm{~cm} \leq \mathrm{DAP}<45 \mathrm{~cm})$ e 26 indivíduos/ha no estoque de exploração (DAP $\geq 45 \mathrm{~cm}$ ).

Das 73 espécies identificadas (Quadro 3), 46 apresentaram densidade absoluta $\geq 1$. Na categoria das espécies comerciais, Virola surinamensis e Carapa guianensis foram as produtoras de madeira de maior destaque, assim como a não-madeireira Euterpe oleracea. Essas três espécies representaram 93,2\% dos indivíduos das espécies desta categoria.

$\mathrm{Na}$ categoria das espécies potenciais destacaram-se Symphonia globulifera, Pentaclethra macroloba, Terminalia dichotoma e Platymiscium trinitatis, compreendendo $91,3 \%$ do total de indivíduos. Quanto à categoria das não-comerciais, de um total de 54 espécies destacaram-se 18 com densidade relativa $\geq 1$, o que corresponde a $82,8 \%$ da densidade absoluta desta categoria de uso. Deste modo, das 73 espécies computadas na amostra, $37(50,7 \%)$ foram consideradas raras, de acordo com o critério de Kageyama \& Gandara (1993).

A dominância da categoria das comerciais referiuse às mesmas espécies de maior densidade; esta situação também ocorreu para a categoria das potenciais. Com relação à categoria das não-comerciais verificou-se que, das 18 que apresentaram densidade relativa $\geq 1$, Inga alba, Gustavia augusta, Tachigalia mymercophila, Cecropia palmata e Miconia ceramicarpa apresentaram dominância relativa $<1$, o que impossibilita a indicação destas espécies para manejo.

Verificou-se que todas as espécies das três categorias de uso consideradas, com DR $\geq 1$ e DoR $\geq 1$, estavam distribuídas em mais de $70 \%$ da área amostrada, à exceção de Platymiscium trinitatis, Allantoma lineata, Pterocarpus amazonicus e Pterocarpus officinalis. 
Quadro 3 - Relação das espécies em ordem decrescente de IVIAE, com suas respectivas famílias, inventariadas em floresta de várzea baixa, propriedade florestal da EMAPA, município de Afuá, Pará. Em que $\mathrm{GE}=$ grupo ecológico $(\mathrm{P}=$ pioneira, $\mathrm{CL}=$ clímax exigente de luz e $\mathrm{CS}=$ clímax tolerante à sombra); $\mathrm{n}=$ número de indivíduos por hectare; $\mathrm{DR}=$ densidade relativa; DoR = dominância relativa; $\mathrm{FR}=$ freqüência relativa; IVI = índice de valor de importância; PSR = posição sociológica relativa; IVIA = índice de valor de importância ampliado; QFR = qualidade de fuste relativa; IVIAE = Índic ede valor de importância ampliado e econômico; $\mathrm{GU}=$ grupo de uso $(\mathrm{C}=$ comerciais, $\mathrm{P}=$ potenciais e $\mathrm{NC}=$ não-comerciais $)$ e $\mathrm{PDE}=$ padrão de distribuição espacial $(\mathrm{AL}=$ aleatório e $\mathrm{AG}=$ agregado)

Table 3 - List of species in decreasing IVIAE order with their respective botanical families, sampled at the low floodplain forest, EMAPA forestlands, Afuá, Pará. Where: $G E=$ ecological group $(P=$ pioneer, $C L=$ light demanding and $C S=$ shade tolerant); $n=$ number of individuals per hectare; $D R=$ relative density; $D o R=$ relative dominance; $F R=$ relative frequency; $I V I=$ importance value index; PSR = relative sociological position; IVIA = amplified importance value index; $Q F R=$ relative quality of trunk; IVIAE = amplified and economic importance value index; $G U=$ use category $(C=$ commercial, $P=$ potential and $N C=$ non-commercial $) ; P D E=$ spatial distribution pattern $(A L=$ randomized and $A G=$ clustering $)$

\begin{tabular}{|c|c|c|c|c|c|c|c|c|c|c|c|c|c|c|}
\hline No- & Espécie & Família & GE & $\mathrm{n}$ & \begin{tabular}{|l|l|l|l|l|} 
DR \\
\end{tabular} & DoR & FR & IVI & PSR & IVI & 2FR & VIAE & $\mathrm{GU}$ & PDE \\
\hline 1 & Virola surinamensis Warb. & Myristicaceae & $\mathrm{CL}$ & 42,7 & 12,0 & 15,5 & 3,0 & 30,5 & 10,8 & 41,3 & 15,7 & 57,0 & $\mathrm{C}$ & $\mathrm{AG}$ \\
\hline 2 & Symphonia globulifera L. f. & Guttiferae & CL & 41,9 & {$[11,7$} & 13,0 & 2,8 & 27,5 & 11,7 & 39,2 & 14,2 & 53,3 & $\mathrm{P}$ & $\mathrm{AG}$ \\
\hline 3 & Eschweilera coriacea Mart. ex Berg & Lecythidaceae & CL & 24,0 & 6,5 & 9,3 & 3,0 & 18,8 & 5,9 & 24,7 & 9,2 & 33,9 & $\mathrm{NC}$ & $\mathrm{AL}$ \\
\hline 4 & Pentaclethra macroloba Kuntze & Leguminosae Mimosoideae & $\mathrm{CS}$ & 20,8 & 5,8 & 4,5 & 3,0 & 13,3 & 6,7 & 20,0 & 4,7 & 24,7 & $\mathrm{P}$ & $\mathrm{AG}$ \\
\hline 5 & Astrocaryum murumuru Mart. & Arecaceae & CL & 33,0 & 9,2 & 3,2 & 3,0 & 15,4 & 5,7 & 21,1 & 0,0 & 21,1 & $\mathrm{NC}$ & $\mathrm{AL}$ \\
\hline 6 & Inga edulis Mart. & Leguminosae Mimosoideae & $\mathrm{CL}$ & 15,1 & 4,2 & 3,0 & 3,0 & 10,3 & 5,1 & 15,4 & 4,5 & 19,8 & $\mathrm{NC}$ & $\mathrm{AG}$ \\
\hline 7 & Carapa guianensis Aubl. & Meliaceae & CL & 12,3 & 3,5 & 5,1 & 3,0 & 11,5 & 3,8 & 15,3 & 4,2 & 19,6 & C & $\mathrm{AG}$ \\
\hline 8 & Terminalia dichotoma G. Mey. & Combretaceae & $\mathrm{CL}$ & 11,1 & 3,1 & 4,8 & 2,5 & 10,4 & 3,3 & 13,8 & 3,9 & 17,7 & $\mathrm{P}$ & $\mathrm{AG}$ \\
\hline 9 & Licania macrophylla Benth. & Chrysobalanaceae & CL & 9,8 & 2,7 & 3,2 & 2,3 & 8,2 & 3,1 & 11,3 & 3,6 & 14,9 & $\mathrm{NC}$ & $\mathrm{AG}$ \\
\hline 10 & Euterpe oleracea Mart. & Arecaceae & $\mathrm{CL}$ & 15,4 & 4,3 & 1,2 & 2,9 & 8,4 & 4,9 & 13,3 & 0,0 & 13,3 & $\mathrm{C}$ & $\mathrm{AG}$ \\
\hline 11 & Crudia bracteata Benth. & Leguminosae Caesalpinioideae & $\mathrm{CS}$ & 10,2 & 2,9 & 1,9 & 2,8 & 7,4 & 3,1 & 10,6 & 2,7 & 13,3 & $\mathrm{NC}$ & $\mathrm{AG}$ \\
\hline 12 & Platymiscium trinitatis Benth. & nosae Papilionoideae & $\mathrm{CL}$ & 8,4 & 2,4 & 2,8 & 1,6 & 6,7 & 2,4 & 9,1 & 2,9 & 12,0 & $\mathrm{P}$ & $\mathrm{AG}$ \\
\hline 13 & Mouriria grandiflora DC. & Melast & $\mathrm{CS}$ & 7,9 & 2,2 & 1,8 & 2,6 & 6,6 & 2,3 & 9,0 & 2,1 & 11,1 & $\mathrm{NC}$ & $\mathrm{AG}$ \\
\hline 14 & Protium spruceanum Engl. & Burs & $\mathrm{CS}$ & 7,9 & 2,2 & 1,3 & 2,4 & 5,9 & 2,4 & 8,4 & 2,5 & 10,8 & $\mathrm{NC}$ & $\mathrm{AG}$ \\
\hline 15 & Hevea brasiliensis Müll. Arg & Eupho & CS & 5,6 & 1,6 & 2,4 & 3,0 & 7,0 & 1,6 & 8,6 & 1,8 & 10,4 & $\mathrm{NC}$ & $\mathrm{AL}$ \\
\hline 16 & Allantoma lineata Miers & Lecyt & $\mathrm{CL}$ & 6,4 & 1,8 & 2,2 & $1,1]$ & 5,0 & $2,1]$ & 7,1 & 2,2 & 9,3 & NC & $\mathrm{AL}$ \\
\hline 17 & Inga alba Willd. & Legu & CL & 5,4 & 1,5 & 0,8 & 2,8 & 5,0 & 1,7 & 6,7 & 1,7 & 8,4 & $\mathrm{NC}$ & $\mathrm{AL}$ \\
\hline 18 & Parinari & aceae & $\mathrm{CS}$ & 2,8 & 0,8 & 3,3 & 2,2 & 6,2 & 0,8 & 7,0 & 1,0 & 8,0 & $\mathrm{NC}$ & $\mathrm{AL}$ \\
\hline 19 & osa Benth. & e Caesa & $\mathrm{CS}$ & 4,2 & 1,2 & 2,2 & 2,2 & 5,5 & 1,5 & 7,0 & 1,0 & 8,0 & $\mathrm{NC}$ &  \\
\hline 20 & Gustavt & Lecyt & $\mathrm{CS}$ & 5,8 & 1,6 & 0,7 & 2,5 & 4,8 & 1,0 & 5,9 & 1,8 & 7,6 & $\mathrm{NC}$ & $\mathrm{AG}$ \\
\hline 21 & Tachigalia my & ae Caesalpinic & $\mathrm{CS}$ & 4,7 & 1,3 & 0,6 & 2,6 & 4,5 & 1,5 & 6,0 & 1,5 & 7,5 & $\mathrm{NC}$ & $\mathrm{AG}$ \\
\hline 22 & Cecropia palmata Willd. & Cecro & PI & 4,5 & 1,3 & 0,7 & 2,4 & 4,4 & 1,6 & 6,0 & 1,5 & 7,5 & NC & $\mathrm{AG}$ \\
\hline 23 & Pterocarpus amazonicus Huber & Leguminosae Papilionoideae & $\mathrm{CL}$ & 3,8 & 1,1 & 1,7 & 2,0 & 4,8 & 1,2 & 6,0 & 0,9 & 6,9 & $\mathrm{NC}$ & $\mathrm{AG}$ \\
\hline 24 & Miconia ceramicarpa Cogn. & ataceae & $\mathrm{CS}$ & 4,0 & 1,1 & 0,5 & 2,0 & 3,7 & 1,1 & 4,8 & 1,2 & 6,0 & $\mathrm{NC}$ & $\mathrm{AG}$ \\
\hline 25 & Pterocarpus officinalis Jacq. & sae Papilionoideae & CL & 3,9 & 1,1 & 1,0 & 1,6 & 3,7 & 1,3 & 4,9 & 0,9 & 5,8 & $\mathrm{NC}$ & $\mathrm{AG}$ \\
\hline 26 & Hymenaea oblongifolia Huber & Leguminosae Caesalpinioideae & CL & 2,3 & 0,7 & 1,6 & 2,0 & 4,3 & 0,5 & 4,7 & 0,9 & 5,6 & $\mathrm{C}$ & $\mathrm{AG}$ \\
\hline 27 & Licania heteromorpha Benth. & 'Chrysobalanaceae & CL & 3,2 & 0,9 & 0,5 & 1,8 & 3,2 & 1,0 & 4,2 & 1,2 & 5,4 & $\mathrm{NC}$ & $\mathrm{AL}$ \\
\hline 28 & Apeiba burchellii Sprague & Tiliaceae & CL & 2,8 & 0,8 & 0,5 & 1,8 & 3,1 & 0,9 & 4,0 & 1,0 & 5,0 & $\mathrm{NC}$ & $\mathrm{AG}$ \\
\hline 29 & Rheedia macrophylla Planch. \& Triana & Guttif & $\mathrm{CL}$ & 2,6 & 0,7 & 0,4 & 1,8 & 2,9 & 0,8 & 3,7 & 1,0 & 4,7 & $\mathrm{NC}$ & $\mathrm{AL}$ \\
\hline 30 & Caraipa grandiflora Mart. & Gutti & $\mathrm{CS}$ & 3,1 & 0,9 & 0,3 & 1,6 & 2,7 & 0,8 & 3,5 & 1,0 & 4,5 & $\mathrm{P}$ & $\mathrm{AL}$ \\
\hline 31 & Mora paraensis Ducke & Leguminosae Caesalpinioideae & $\mathrm{CS}$ & 1,8 & 0,5 & 1,4 & 1,4 & 3,3 & 0,5 & 3,8 & 0,7 & 4,5 & $\mathrm{P}$ & $\mathrm{AG}$ \\
\hline 32 & Inga velutina Willd. & Leguminosae Mimosoideae & CL & 2,4 & 0,7 & 0,6 & 1,7 & 2,9 & 0,8 & 3,7 & 0,7 & 4,4 & $\mathrm{NC}$ & $\mathrm{AG}$ \\
\hline 33 & Ficus gomelleira Kunth et Bob & Mora & CL & 1,6 & 0,5 & 1,3 & 1,7 & 3,4 & 0,5 & 4,0 & 0,4 & 4,4 & NC & $\mathrm{AG}$ \\
\hline 34 & Virola s & Myri & $\mathrm{CL}$ & 2,4 & 0,7 & 0,7 & 1,6 & 2,9 & 0,6 & 3,5 & 0,8 & 4,3 & $\mathrm{C}$ & $\mathrm{AG}$ \\
\hline 35 & igiana Mart. & Anno & CL & 2,2 & 0,6 & 0,3 & 1,6 & 2,5 & 0,6 & 3,1 & 0,6 & 3,7 & $\mathrm{NC}$ & $\mathrm{AG}$ \\
\hline 36 & Licania cf. octandra Kuntze & Chrys & $\mathrm{CS}$ & 1,8 & 0,5 & 0,4 & 1,2 & 2,1 & 0,6 & 2,6 & 0,7 & 3,3 & $\mathrm{NC}$ & $\mathrm{AG}$ \\
\hline 37 & Tapirira guianensis Aubl. & Anaca & PI & 1,4 & 0,4 & 0,5 & 1,2 & 2,1 & 0,5 & 2,5 & 0,5 & 3,0 & $\mathrm{P}$ & $\mathrm{AG}$ \\
\hline 38 & ns Benoist. & Chrysobalanacea & $\mathrm{CS}$ & 1,3 & 0,4 & 0,4 & 1,2 & 2,0 & 0,5 & 2,4 & 0,4 & 2,9 & $\mathrm{NC}$ & $\mathrm{AG}$ \\
\hline 39 & Eugenia brownsbergii Amshoff & Myrtaceae & $\mathrm{CL}$ & 1,6 & 0,5 & 0,2 & 1,2 & 1,9 & 0,5 & 2,4 & 0,5 & 2,9 & $\mathrm{NC}$ & $\mathrm{AG}$ \\
\hline 40 & Sarcaulus brasiliensis (A. DC.) Eym & Sapotaceae & $\mathrm{CS}$ & 1,0 & 0,3 & 0,2 & 1,4 & 1,9 & 0,4 & 2,2 & 0,3 & 2,5 & $\mathrm{NC}$ & $\mathrm{AG}$ \\
\hline 41 & Guarea guidonia (L.) Sleumer & Meliaceae & $\mathrm{CS}$ & 1,4 & 0,4 & 0,3 & 1,1 & 1,7 & 0,3 & 2,1 & 0,4 & 2,5 & $\mathrm{NC}$ & $\mathrm{AG}$ \\
\hline 42 & Taralea oppositifolia Aubl. & Leguminosae Papilionoideae & $\mathrm{CS}$ & 1,0 & 0,3 & 0,3 & 1,0 & 1,6 & 0,4 & 1,9 & 0,3 & 2,2 & $\mathrm{NC}$ & $\mathrm{AL}$ \\
\hline 43 & Sterculia pruriens K. Schum. & Sterculiaceae & CL & 1,0 & $0,3]$ & 0,2 & 1,1 & 1,5 & 0,3 & 1,8 & 0,4 & 2,2 & P & $\mathrm{AG}$ \\
\hline
\end{tabular}

Continua...

Continued... 
Quadro 3, Cont. Table 3, Cont.

\begin{tabular}{|c|c|c|c|c|c|c|c|c|c|c|c|c|c|c|}
\hline jo & Espécie & Família & GE & $\mathrm{n}$ & $\overline{D R}$ & DoR & FR & IVI & PSR & IVIA & $\overline{Q F R} \mathrm{II}$ & IVIAE & $\mathrm{GU}$ & $\mathrm{PDE}$ \\
\hline 44 & Oenocarpus bacaba Mart. & Arecaceae & $\mathrm{CS}$ & 1,2 & 0,3 & 0,2 & 1,1 & 1,6 & 0,3 & 1,9 & 0,0 & 1,9 & $\mathrm{NC}$ & $\mathrm{AG}$ \\
\hline 45 & Vatairea guianensis Aubl. & Leguminosae Papilionoideae & PI & 0,7 & 0,2 & 0,3 & 1,0 & 1,4 & 0,2 & 1,7 & 0,2 & 1,9 & $\mathrm{NC}$ & $\mathrm{AG}$ \\
\hline 46 & Vismia macrophylla H. B. K. & Guttiferae & $\mathrm{PI}$ & 1,0 & 0,3 & 0,1 & 0,6 & 1,0 & 0,3 & 1,3 & 0,3 & 1,5 & $\mathrm{NC}$ & $\mathrm{AG}$ \\
\hline 47 & Indeterminada 1 & & - & 0,5 & 0,1 & 0,3 & 0,7 & 1,1 & 0,1 & 1,2 & 0,2 & 1,4 & $\mathrm{NC}$ & $\mathrm{AL}$ \\
\hline 48 & Nectandra pisi Miq. & Lauraceae & $\mathrm{CL}$ & 0,6 & 0,2 & 0,1 & 0,7 & 1,0 & 0,2 & 1,2 & 0,2 & 1,4 & $\mathrm{NC}$ & $\mathrm{AG}$ \\
\hline 49 & Manicaria saccifera Gaertn. & Areca & $\mathrm{PI}$ & 1,0 & 0,3 & 0,3 & 0,6 & 1,2 & 0,2 & 1,3 & 0,0 & 1,3 & $\mathrm{NC}$ & $\overline{A L}$ \\
\hline 50 & Iryanthera sagotiana (Benth.) Warb. & Myristicace & $\mathrm{CL}$ & 0,5 & 0,1 & 0,1 & 0,7 & 0,9 & 0,2 & 1,1 & 0,2 & 1,3 & $\mathrm{NC}$ & $\mathrm{AG}$ \\
\hline 51 & Eugenia floribunda $\mathrm{H}$. West ex Willd. & Myr & PI & 0,9 & 0,3 & 0,1 & 0,5 & 0,8 & 0,2 & 1,0 & 0,2 & 1,2 & $\mathrm{NC}$ & $\mathrm{AL}$ \\
\hline 52 & Spondias mombin L. & Anac & PI & 0,6 & 0,2 & 0,3 & 0,4 & 0,8 & 0,2 & 1,0 & 0,2 & 1,2 & $\mathrm{NC}$ & $\mathrm{AG}$ \\
\hline 53 & Zygia juruana (Harms) L. Ri & Legu & CL & 0,6 & 0,2 & 0,1 & 0,7 & 0,9 & 0,1 & 1,0 & 0,1 & 1,1 & NC & $\mathrm{AL}$ \\
\hline 54 & Schefflera paraensis Huber & Arali & CL & 0,3 & 0,1 & 0,3 & 0,5 & 0,8 & 0,1 & 0,9 & 0,1 & 1,0 & $\mathrm{NC}$ & $\mathrm{AL}$ \\
\hline 55 & Caryocar villosum Pers. & Cary & CL & 0,4 & 0,1 & 0,2 & 0,4 & 0,7 & 0,1 & 0,8 & 0,1 & 0,9 & $\mathrm{NC}$ & $\mathrm{AG}$ \\
\hline 56 & Caryocar microcarpum Ducke & Caryo & CL & 0,2 & 0,1 & 0,2 & 0,4 & 0,6 & 0,1 & 0,7 & 0,1 & 0,7 & $\mathrm{NC}$ & $\mathrm{AL}$ \\
\hline 57 & nguba Mart. & Bom & CL & 0,2 & 0,1 & 0,4 & 0,2 & 0,6 & 0,0 & 0,7 & 0,1 & 0,7 & C & $\mathrm{AG}$ \\
\hline 58 & Indet & - & $=$ & 0,2 & 0,1 & 0,1 & 0,4 & 0,5 & 0,1 & 0,6 & 0,1 & 0,7 & $\mathrm{NC}$ & $\mathrm{AG}$ \\
\hline 59 & da copaia $\mathrm{D}$. Don & Big & CL & 0 & 0,1 & 0,2 & 0,2 & 0,5 & 0,0 & 0,5 & 0,1 & 0,6 & $\mathrm{C}$ & $\mathrm{AL}$ \\
\hline 60 & Sloanea grandiflora C. E. Sm. & Elae & PI & 0,2 & 0,1 & 0,1 & 0,2 & 0,4 & 0,1 & 0,5 & 0,1 & 0,6 & $\mathrm{NC}$ & $\mathrm{AL}$ \\
\hline 61 & Bombax aquaticum (Aubl.) K. & Bomt & CS & 0,2 & 0,1 & 0,1 & 0,2 & 0,4 & 0,0 & 0,4 & 0,1 & 0,5 & $\mathrm{NC}$ & $\mathrm{AG}$ \\
\hline 62 & Hernandia guianensis Aubl. & Hernandiaceae & CL & 0,2 & 0,1 & 0,1 & 0,2 & 0,4 & 0,1 & 0,4 & 0,1 & 0,5 & $\mathrm{NC}$ & $\mathrm{AL}$ \\
\hline 63 & Couratari guianensis Aubl. & Lecythidaceae & CL & 0,2 & 0,1 & 0,1 & 0,2 & 0,4 & 0,1 & 0,4 & 0,1 & 0,5 & $\mathrm{NC}$ & $\mathrm{AG}$ \\
\hline 64 & Sacoglottis guianensis Benth & Hum & CS & 0,2 & 0,1 & 0,1 & 0,2 & 0,3 & 0,1 & 0,4 & 0,1 & 0,5 & $\mathrm{P}$ & $\mathrm{AL}$ \\
\hline 65 & Ficus & Mor & $\mathrm{C}$ & 0 , & 0,1 & 0 , & 0,2 & 0,3 & 0,1 & 0,4 & 0,1 & 0,4 & $\mathrm{NC}$ & $\mathrm{AG}$ \\
\hline 66 & obilis Müll. Ar & Euph & $\mathrm{CS}$ & 0,2 & 0,1 & 0 , & 0,2 & 0,3 & 0,1 & 0,4 & 0,1 & 0,4 & $\mathrm{NC}$ & $\mathrm{AL}$ \\
\hline 67 & Calophyllum brasiliense Can & Gutti & $\mathrm{CS}$ & 0,2 & 0,1 & 0,1 & 0,2 & 0,3 & 0,0 & 0,4 & 0,1 & 0,4 & $\mathrm{P}$ & $\mathrm{AL}$ \\
\hline 68 & Swartzia acuminata Willd. ex Vog. & Leguminosae Caesalpinioideae & CL & 0,2 & 0,1 & 0,1 & 0,2 & 0,3 & 0,1 & 0,4 & 0,0 & 0,4 & $\mathrm{NC}$ & $\mathrm{AL}$ \\
\hline 69 & Ormosia coccinea Jacks. & Leguminosae Papilionoideae & CL & 0,2 & 0,1 & 0,0 & 0,2 & 0,3 & 0,0 & 0,4 & 0,1 & 0,4 & $\mathrm{NC}$ & $\overline{A L}$ \\
\hline 70 & Ormosia coutinhoi Ducke & Leguminosae Papilionoideae & CL & 0,2 & 0,1 & 0,0 & 0,2 & 0,3 & 0,0 & 0,3 & 0,1 & 0,4 & $\mathrm{P}$ & $\mathrm{AL}$ \\
\hline 71 & Macrolobium a & losae Caesa & $\mathrm{C}$ & 0,1 & 0,0 & 0, & 0,1 & 0,2 & 0,0 & 0,2 & 0,0 & 0,2 & $P$ & $\mathrm{AL}$ \\
\hline 72 & Socrat & Arec & CS & 0,1 & 0,0 & 0,0 & 0,1 & 0,2 & 0,0 & 0,2 & 0,0 & 0,2 & $\mathrm{NC}$ & \\
\hline 73 & Quararibea guianensis Aubl. & Bombacaceae & $\mathrm{CL}$ & 0,1 & 0,0 & 0,0 & 0,1 & 0,2 & 0,0 & 0,2 & 0,0 & 0,2 & $\mathrm{NC}$ & - \\
\hline
\end{tabular}

Para verificar a ocorrência das espécies nos diferentes estratos da floresta, realizou-se a análise da posição sociológica das espécies (PSR) e do IVIA. Esta análise permitiu identificar que entre as quatro espécies que apresentaram maior densidade e dominância no grupo das potenciais apenas Platymiscium trinitatis não apresentou indivíduos no estrato inferior, o que pode vir a comprometer sua participação nos demais estratos, no futuro. Todas as espécies não-comerciais com DR $\geq 1$ e DoR $\geq 1$ foram consideradas para fins de manejo, visto que estavam representadas em todos os estratos da floresta.

Em termos de qualidade de fuste e IVIAE, observouse que para a categoria das comerciais Virola surinamensis e Carapa guianensis, além de Euterpe oleracea, apresentaram bons índices e, portanto, foram incluídas no grupo das espécies a serem manejadas. Já na categoria das potenciais as espécies eleitas foram: Symphonia globulifera, Pentaclethra macroloba e Terminalia dichotoma, enquanto na categoria das não comerciais as espécies credenciadas foram Eschweilera coriacea,

R. Árvore, Viçosa-MG, v.26, n.3, p.311-319, 2002
Swartzia racemosa, Inga edulis, Mouriria grandiflora, Licania macrophylla, Allantoma lineata, Pterocarpus amazonicus, Pterocarpus officinalis e Parinari excelsa; porém as excluídas deste grupo foram Hevea brasiliensis, por ser uma espécie proibida de corte (Instituto... IBAMA, 1995), Crudia bracteata e Protium spruceanum, por não apresentarem indivíduos em todos os estratos da floresta.

As espécies com maior índice de valor de importância ampliado e econômico - IVIAE - foram: Virola surinamensis, Symphonia globulifera, Eschweilera coriacea, Pentaclethra macroloba, Astrocaryum murumuru, Inga edulis, Carapa guianensis, Terminalia dichotoma, Licania macrophylla e Euterpe oleracea (Quadro 3). Gama (2000) verificou que todas essas espécies ocorreram na regeneração natural e que elas são igualmente aproveitadas para produção de madeira ou de produtos não-madeireiros. Portanto, o IVIAE é uma variável indicada para diagnóstico do potencial produtivo de florestas nativas. 
As análises evidenciaram que as espécies amostradas têm potencial para produzir 54 toras/ha $(59,3 \%$ toras de $1^{\mathrm{a}}, 25,9 \%$ toras de $2^{\mathrm{a}}$ e $14,8 \%$ toras de $\left.3^{\mathrm{a}}\right)$, o que representa um volume em torno de $60,00 \mathrm{~m}^{3} / \mathrm{ha}$, que poderá ser colhido de $20 \mathrm{em} 20$ anos, gerando uma receita potencial final de toras de US\$ 501,7/ha (Quadro 4).

A floresta estudada foi explorada de 1955 até 1992 - período de extração intensiva de Virola surinamensis, Carapa guianensis e palmito de Euterpe oleracea.
Apesar deste histórico, a avaliação das espécies comerciais nesse ambiente demonstrou que Virola surinamensis apresentou o maior número de toras em relação ao total (50,5\%), em que $35,1 \%$ destes estavam concentrados em toras de $1^{\mathrm{a}}$ classe, conforme a classificação local. Carapa guianensis também foi uma espécie de uso comercial que se destacou, representando $28,8 \%$ das toras desta categoria, em que 50,4\% eram de toras de $1 \stackrel{\text { a }}{\text {. Estas }}$ duas espécies representaram, assim, 79,3\% do número de toras desta categoria.

Quadro 4 - Número de toras por hectare (NTila), preço médio das toras (PMTe receita potencial (RP) por classe de tora (T) propriedade florestal da EMAPA, município de Afuá, Pará

Table 4 - Number of boles per hectare (NT/ha), medium price of boles (PMT) and potential income (RP) by bole class (T), at EMAPA forestlands, Afuá, Pará

\begin{tabular}{|c|c|c|c|c|c|c|c|c|c|c|c|c|}
\hline \multirow{2}{*}{ № } & \multirow{2}{*}{ Uso/Espécie } & \multicolumn{4}{|c|}{ NT (ha) } & \multicolumn{3}{|c|}{ PMT (US\$) } & \multicolumn{4}{|c|}{ RP (US\$/ha) } \\
\hline & & $\mathrm{T}_{1}$ & $\mathrm{~T}_{2}$ & $\mathrm{~T}_{3}$ & Total & $\mathrm{T}_{1}$ & $\mathrm{~T}_{2}$ & $\mathrm{~T}_{3}$ & $\mathrm{~T}_{1}$ & $\mathrm{~T}_{2}$ & $\mathrm{~T}_{3}$ & Total \\
\hline \multicolumn{13}{|c|}{ Comerciais } \\
\hline 1 & Virola surinamensis & 3,0 & 2,8 & 2,7 & 8,5 & 16,7 & 8,3 & 5,0 & 49,7 & 23,3 & 13,6 & 86,6 \\
\hline 2 & Carapa guianensis & 2,4 & 1,4 & 1,0 & 4,8 & 16,7 & 8,3 & 5,0 & 40,7 & 12,0 & 4,8 & 57,4 \\
\hline 3 & Hymenaea oblongifolia & 1,8 & 0,5 & 0,2 & 2,4 & 17,8 & 10,6 & 6,1 & 32,0 & 5,1 & 1,0 & 38,1 \\
\hline 4 & Virola sp. & 0,0 & 0,0 & 0,0 & 0,0 & 16,7 & 8,3 & 5,0 & 0,0 & 0,0 & 0,0 & 0,0 \\
\hline 5 & Bombax munguba & 0,6 & 0,0 & 0,0 & 0,6 & 8,3 & 4,4 & 0,0 & 5,0 & 0,0 & 0,0 & 5,0 \\
\hline 6 & Jacaranda copaia & 0,2 & 0,2 & 0,0 & 0,4 & 11,1 & 6,7 & 4,4 & 2,2 & 1,6 & 0,0 & 3,8 \\
\hline & Subtotal & 8,0 & 5,0 & 3,8 & 16,8 & & & & 129,6 & 42,0 & 19,4 & 190,9 \\
\hline \multicolumn{13}{|c|}{ Potenciais } \\
\hline 1 & Symphonia globulifera & 2,5 & 1,6 & 1,0 & 5,1 & 10,2 & 5,9 & 2,9 & 25,4 & 9,5 & 2,8 & 37,8 \\
\hline 3 & Terminalia dichotoma & 2,6 & 1,1 & 0,6 & 4,3 & 10,2 & 5,9 & 2,9 & 26,5 & 6,7 & 1,7 & 34,8 \\
\hline 4 & Platymiscium trinitatis & 0,9 & 0,3 & 0,3 & 1,5 & 10,2 & 5,9 & 2,9 & 8,8 & 1,9 & 0,9 & 11,6 \\
\hline 2 & Pentaclethra macroloba & 0,0 & 0,0 & 0,0 & 0,0 & 10,2 & 5,9 & 2,9 & 0,0 & 0,0 & 0,0 & 0,0 \\
\hline 5 & Outras potenciais & 1,5 & 0,5 & 0,1 & 2,0 & 10,2 & 5,9 & 2,9 & 14,9 & 2,8 & 0,2 & 17,9 \\
\hline & Subtotal & 7,4 & 3,5 & 1,9 & 12,9 & & & & 75,6 & 20,9 & 5,7 & 102,1 \\
\hline \multicolumn{13}{|c|}{ Não-comerciais } \\
\hline 1 & Eschweilera coriacea & 3,9 & 2,0 & 1,5 & 7,5 & 10,2 & 5,9 & 2,9 & 40,1 & 11,8 & 4,5 & 56,5 \\
\hline 2 & Inga edulis & 0,4 & 0,2 & 0,0 & 0,5 & 10,2 & 5,9 & 2,9 & 3,7 & 0,9 & 0,0 & 4,6 \\
\hline 3 & Licania macrophylla & 1,4 & 0,5 & 0,1 & 2,0 & 10,2 & 5,9 & 2,9 & 14,7 & 2,8 & 0,2 & 17,7 \\
\hline 4 & Mouriria grandiflora & 0,1 & 0,1 & 0,0 & 0,2 & 10,2 & 5,9 & 2,9 & 1,0 & 0,5 & 0,0 & 1,5 \\
\hline 5 & Allantoma lineata & 0,7 & 0,6 & 0,2 & 1,4 & 10,2 & 5,9 & 2,9 & 6,7 & 3,3 & 0,5 & 10,5 \\
\hline 6 & Parinari excelsa & 4,2 & 0,6 & 0,2 & 5,0 & 10,2 & 5,9 & 2,9 & 42,4 & 3,8 & 0,5 & 46,6 \\
\hline 7 & Swartzia racemosa & 0,8 & 0,3 & 0,0 & 1,1 & 10,2 & 5,9 & 2,9 & 8,3 & 1,9 & 0,0 & 10,2 \\
\hline 8 & Pterocarpus amazonicus & 0,9 & 0,0 & 0,0 & 0,9 & 10,2 & 5,9 & 2,9 & 9,4 & 0,0 & 0,0 & 9,4 \\
\hline 9 & Pterocarpus officinalis & 0,2 & 0,1 & 0,0 & 0,3 & 10,2 & 5,9 & 2,9 & 2,1 & 0,5 & 0,0 & 2,5 \\
\hline 10 & Outras não-comerciais & 4,1 & 1,0 & 0,2 & 5,4 & 10,2 & 5,9 & 2,9 & 42,2 & 6,2 & 0,7 & 49,1 \\
\hline & Subtotal & 16,7 & 5,4 & 2,2 & 24,3 & & & & 170,4 & 31,8 & 6,4 & 208,6 \\
\hline & Total & 32 & 14 & 8 & 54 & & & & 375,6 & 94,4 & 31,7 & - \\
\hline & RPT (US\$) & & & & & & & & & & & 501,7 \\
\hline
\end{tabular}

Em que: $\mathrm{T}_{1}=$ tora de $1^{\mathrm{a}}, \mathrm{T}_{2}=$ tora de $2^{\mathrm{a}}, \mathrm{T}_{3}=$ tora de $3^{\underline{a}}$ e $\mathrm{RPT}=$ receita potencial de tora.

Where: $T_{1}=1^{\text {st }}$ class bole, $T_{2}=2^{\text {nd }}$ class bole and $T_{3}=3^{\text {rd }}$ class bole. 
Quanto às espécies potenciais, Symphonia globulifera foi a que apresentou o maior número de toras $(39,3 \%)$ em relação ao total, com $49,4 \%$ concentradas na classe de tora de $1 \stackrel{\mathrm{a}}{\text {, }}$, seguida por Terminalia dichotoma, que contribuiu com $33,3 \%$ do total de toras, das quais $60,7 \%$ foram toras de 1 a. Juntas estas duas espécies corresponderam a $72,6 \%$ das toras desta categoria. Com referência às não-comerciais, as espécies que contribuíram com os maiores porcentuais de toras em relação ao total foram apenas Eschweilera coriacea $(30,8 \%)$ e Parinari excelsa (20,4\%). Estas espécies apresentaram, respectivamente, 52,2 e $83,9 \%$ de toras de $1^{\mathfrak{a}}$, correspondendo a $51,2 \%$ do número de toras desta categoria.

Essa avaliação destacou que as espécies comerciais, potenciais e não-comerciais contribuíram com 38,1, 20,4 e $41,5 \%$ da receita potencial de toras, respectivamente, e que, portanto, houve maior contribuição das espécies nãocomerciais no que se refere ao número de toras aproveitáveis. A inclusão dessas espécies na lista de exploração poderá incrementar substancialmente a receita potencial de toras.

A produção potencial anual de palmito (Quadro 5) indica a possibilidade de obtenção de uma receita de até US\$ 68,5/ha. Portanto, foi possível verificar que a receita potencial final, considerando apenas o estoque de exploração de madeira e de palmito, foi de US\$ 570,20/ha.

Quadro 5-Receita potencial do palmito em floresta de várzea baixa, propriedade florestal da EMAPA, município de Afuá, Pará

Table 5-Heart-palm potential income in a low floodplain forest at EMAPA forestlands, Afuá, Pará

\begin{tabular}{|l|c|c|c|}
\hline \multicolumn{1}{|c|}{ Classe de Palmito } & $\begin{array}{c}\text { Quantidade de } \\
\text { Palmitos/ha }\end{array}$ & $\begin{array}{c}\text { Preço } \\
(\text { US\$ })\end{array}$ & $\begin{array}{c}\text { Receita } \\
\text { Potencial } \\
(\text { US\$/ha })\end{array}$ \\
\hline $1^{\text {a }}(\mathrm{DAP} \geq 15 \mathrm{~cm})$ & 61,67 & 0,17 & 10,48 \\
$2^{\mathrm{a}}(10 \mathrm{~cm} \leq \mathrm{DAP}<15 \mathrm{~cm})$ & 528,00 & 0,11 & 58,00 \\
\hline \multicolumn{1}{|c|}{ Total } & 589,67 & & 68,48 \\
\hline
\end{tabular}

\section{CONCLUSÃO}

Apesar de ter sido intensamente explorada até 1992, a floresta secundária de várzea baixa estudada apresentou um estoque de exploração que pode ser aproveitado economicamente. As espécies com maior valor de

R. Árvore, Viçosa-MG, v.26, n.3, p.311-319, 2002 importância ampliado e econômico foram Virola surinamensis, Symphonia globulifera, Eschweilera coriacea, Pentaclethra macroloba, Astrocaryum murumuru, Inga edulis, Carapa guianensis, Terminalia dichotoma, Licania macrophylla e Euterpe oleracea. A produção potencial de toras foi de 54 toras/ha, enquanto a de palmito foi de 589,7 palmitos/ha, o que gerou uma receita potencial final de US\$570,20/ha. A análise da vegetação é uma ferramenta fundamental para gerar informações sobre a estrutura da floresta e dar subsídios ao planejamento de uma exploração florestal sustentada. A valoração do estoque de exploração, como instrumento básico de avaliação monetária, permitiu a previsão dos lucros brutos possíveis de serem obtidos com o corte de toras de espécies de interesse comercial, além do que indicou que a inclusão de outras categorias de espécies poderá incrementar a receita potencial final do produtor florestal.

\section{AGRADECIMENTOS}

Aos trabalhadores da EMAPA-Projeto Santana, pela valiosa ajuda no trabalho de campo. Ao professor Manoel Tourinho, pelo apoio e pelos incentivos no dia-a-dia. À Faculdade de Ciências Agrárias do Pará (FCAP) e à CAPES, pela bolsa de mestrado concedida. À Exportadora de Madeiras do Pará Ltda. - EMAPA, pelo auxílio financeiro a este estudo.

\section{REFERÊNCIAS BIBLIOGRÁFICAS}

ANDERSON, A. B. et al. Forest management patterns in the floodplain of the Amazon Estuary. Conservation Biology, v. 9, n. 1, p. 47-61, 1995.

ARAÚJO, A. P.; JORDY FILHO, S.; FONSECA, W. N. A vegetação da Amazônia brasileira. In: SIMPÓSIO DO TRÓPICO ÚMIDO, 1., 1984, Belém. Anais... Belém: EMBRAPA-CPATU, 1986. p. 135-152. (EMBRAPACPATU. Documentos, 36)

BARROS, P. L. C. Estudo fitossociológico de uma floresta tropical úmida no planalto de Curuá-Una, Amazônia brasileira. Curitiba: Universidade federal do Paraná, 1986. 147 p. Tese (Doutorado em Engenharia Florestal) - Universidade federal do Paraná, 1986.

CAMPBELL, D. G. et al. Quantitative ecological inventory of terra firme and várzea tropical forest on the rio Xingu, Brazilian Amazon. Brittonia, v. 38, n. 4, p. 369-393, 1986. 
COSTA-NETO, F. Subsídios técnicos para um plano de manejo sustentado em áreas de cerrado. Viçosa, MG: Universidade Federal de Viçosa, 1990. 142 p. Dissertação (Mestrado em Ciências Florestais) - Universidade Federal de Viçosa, 1990.

CURTIS, J. T.; McINTOSH, R. P. An upland forest continuum in the prairie forest border region of Wisconsin. Ecology, v. 32, p. 476-496, 1951.

FAO. Manual de inventário florestal con especial referencia a los bosques mistos tropicales. Roma: FAO, 1974. $195 \mathrm{p}$.

FERREIRA, R. L. C. Análise estrutural da vegetação da Estação Florestal da Experimentação de Açú - RN, como subsídio básico para o manejo florestal. Viçosa, MG: Universidade Federal de Viçosa, 1988. 91 p. Dissertação (Mestrado em Ciências Florestais) - Universidade Federal de Viçosa, 1988.

FINOL, U. H. Nuevos parametros a considerarse en el analises estructural de las Selvas Virgines tropicais. Revista Florestal Venezolana, v. 14, n. 21, p. 29-42, 1971.

GAMA, J. R. V. Estudo da regeneração natural em floresta de várzea como base para o manejo florestal. Lavras: Universidade Federal de Lavras, 2000. 114 p. Dissertação (Mestrado em Engenharia Florestal) Universidade Federal de Lavras, 2000.

INSTITUTO BRASILEIRO DO MEIO AMBIENTE E DOS RECURSOS NATURAIS RENOVÁVEIS - IBAMA. Portaria no 48 de 10 de julho de 1995. Brasília: MMA/ IBAMA, 1995. $41 \mathrm{p}$.

JARDIM, F. C. S.; HOSOKAWA, R. T. Estrutura da floresta equatorial úmida da Estação Experimental de Silvicultura Tropical do INPA. Acta Amazonica, v. 16/17, p. 411-508, 1986/87.

KAGEYAMA, P.; GANDARA, F. B. Dinâmica de populações de espécies arbóreas: implicações para o manejo e a conservação. In: SIMPÓSIO DE ECOSSISTEMAS DA COSTA BRASILEIRA, 3., 1993, São Paulo. Anais... São Paulo: 1993. $12 \mathrm{p}$.

LAMPRECHT, H. Ensayo sobre la estructura floristica de la parte sur-oriental del Bosque Universitario "El Caimital", Estado Barinas. Revista Forestal Venezolana, v. 7, n. 10/11, p. 77-119, 1964.

LISBOA, P. L. B.; TEREZO, E. F. M.; SILVA, J. C. A. Madeiras amazônicas: considerações sobre exploração, extinção de espécies e conservação. Boletim do Museu Paraense Emílio Goeldi, Série Botânica, v. 7, n. 2, p. 521542, 1991
LUDWIG, J. A.; REYNOLDS, J. F. Statistical ecology: a primer on methods and computing. New York: John Wiley \& Sons, 1988. 337 p.

MACEDO, D. S. M. S. Estrutura e manejo de uma floresta de várzea do estuário amazônico. Piracicaba: Escola Superior de Agricultura "Luiz de Queiroz", 1996. 117 p. Dissertação (Mestrado em Ciências Florestais) Escola Superior de Agricultura "Luiz de Queiroz", 1996.

MARISCAL-FLORES, E. J. Potencial produtivo e alternativas de manejo sustentável de um fragmento de Mata Atlântica secundária, Município de Viçosa, Minas Gerais. Viçosa, MG: Universidade Federal de Viçosa, 1993. 165 p. Dissertação (Mestrado em Ciência Florestal) - Universidade Federal de Viçosa, 1993.

MELlO, J. M. Análise comparativa de procedimentos amostrais em um remanescente de floresta nativa no município de Lavras (MG). Lavras: Universidade Federal de Lavras, 1995. 88p. Dissertação (Mestrado em Engenharia Florestal) - Universidade Federal de Lavras, 1995 .

MUELlER-DOMBOis, D.; EllenBerG, G. H. Aims and methods of vegetation ecology. New York: Willey y Sons, 1974. 546 p.

NAPPO, M. E. Inventário florístico e estrutural da regeneração natural no sub-bosque de povoamentos homogêneos de Mimosa scabrella Bentham, implantados em áreas mineradas, em Poços de Caldas, Minas Gerais. Lavras: Universidade Federal de Lavras, 1999. 87 p. Dissertação (Mestrado em Engenharia Florestal) Universidade Federal de Lavras, 1999.

OLIVEIRA-FILHO, A. T. Estudos ecológicos da vegetação como subsídio para programas de revegetação com espécies nativas: uma proposta metodológica. Cerne, v. 1, n. 1, p. 64-72, 1994.

SOUZA, A. L. Manejo florestal. Viçosa, MG:

Universidade Federal de Viçosa, 1990. (Notas de Aula).

SUDAM/PROJETO DE HIDROLOGIA E CLIMATOLOGIA DA AMAZÔNIA. Atlas Climatológico da Amazônia Brasileira. Belém: SUDAM, 1984. 125 p.

SWAINE, M. D.; WHITMORE, T. C. On the definition of ecological species groups in tropical rain forests. Vegetatio, v. 75 , p. $81-86,1988$.

VIEIRA, L. S. Manual da ciência do solo: com ênfase aos solos tropicais. São Paulo: Agronômica Ceres, 1988. $464 \mathrm{p}$. 